\title{
Editorial: Introduction to the 33rd Annual Gallery of Fluid Motion (Boston, Massachusetts, USA, 2015)
}

\author{
Andrzej Herczyński ${ }^{1}$ and John W. M. Bush ${ }^{2}$ \\ ${ }^{1}$ Boston College, Chestnut Hill, Massachusetts 02467, USA \\ ${ }^{2}$ Massachusetts Institute of Technology, Cambridge, Massachusetts 02139, USA \\ (Received 18 August 2016; published 12 September 2016)
}

DOI: 10.1103/PhysRevFluids.1.050001

The 33rd Gallery of Fluid Motion (GFM) was held November 22-24, 2015, during the 68th annual meeting of the Division of Fluid Dynamics of the American Physical Society (APS) in Boston. As usual, the GFM had two categories, posters and videos, which could be uploaded on the meeting's website (http://gfm.aps.org/meetings/dfd-2015). The deadline for submissions was October 6, 2015. In total, we received 52 posters and 78 videos. (In 2014 these numbers were 70 and 75, respectively, and in 2013, 60 and 68, respectively). Submissions were received from 22 countries and ranged over a broad spectrum of fluid mechanics topics, with the largest number of entries (26\%) on the subject of drops and bubbles (Fig. 1).

All posters and videos were displayed in Hall D of the Haynes Convention Center in Boston, the venue of the meeting. Videos were looped on multiple display screens in a separated area of the hall. For the first time since the inception of GFM in 1992, all video entries were available for viewing online in advance of the meeting at a dedicated site (http://gfm.aps.org).

To select winners of the 2015 Gallery, two panels of judges were assembled from distinguished past contributors who have not served in this capacity in recent years, one for posters (three judges) and one for videos (6 judges). Judges were asked to render their verdicts "primarily on the basis of aesthetic appeal, novelty and creativity, on the grounds that technical merit and scientific content are amply rewarded elsewhere. First and foremost [...] ratings [were to] reward those entries that celebrate the art and beauty of fluids." Poster judges were asked to create a ranked list of the ten entries they deemed the best. Videos were judged in two stages. In the first stage, each judge ranked about 25 entries on the scale of $1-5$, with every video evaluated by two judges. In the second stage, the 12 leading entries were ranked by each judge. In this manner, five winning posters and seven winning videos were identified. The top three winners in each category were designated the Milton van Dyke Award winners. The winning GFM posters and videos are listed below.

\section{Milton van Dyke Award poster winners}

- Drop floating on a granular raft. Etienne Jambon-Puillet and Suzie Protiere.

- An emerging string of fluid pearls. Seong Jin Kim, Sunghwan Jung, Minsu Jang, and Soong Ho Um.

- Variable density vortex ring dynamics in sharply stratified ambient fluids. Roberto Camassa, Richard McLaughlin, Pierre-Yves Passaggia, David Holz, Keith Mertens, and Claudio Viotti.

\section{Milton van Dyke Award video winners}

- The tightrope dancer bubbles. Alexis Duchesne and Charles Dubois.

- The merger of a bubble and a soap film. Giuseppe Pucci, Daniel M. Harris, Victor Prost, Julio Quintela Casal, and John W. M. Bush.

- Placing Marangoni instabilities under arrest. M. Saad Bhamla and Gerald G. Fuller.

\section{Gallery of Fluid Motion poster winners}

- Pollen, water, and wind - chaotic mixing in a puddle of water. Kaare H. Jensen.

- Erosion patterns on dissolving solids. Caroline Cohen, Julien Derr, Michael Berhanu, and Sylvain Courrech du Pont. 


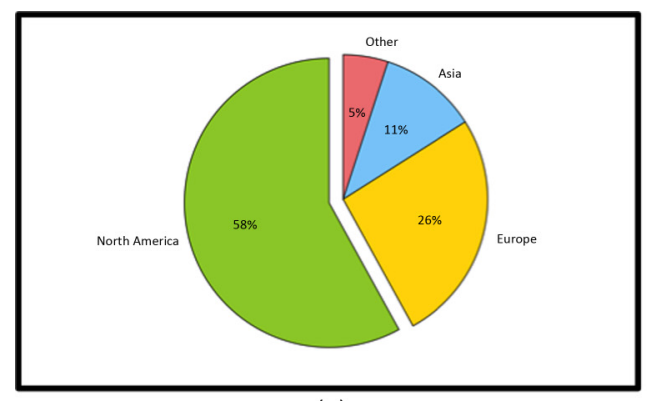

(a)

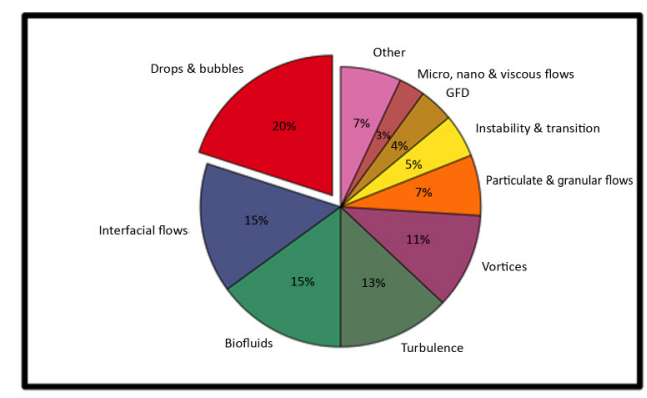

(b)

FIG. 1. (a): Regional distribution of contributed posters and videos. (b): Topical distribution of contributed posters and videos.

\section{Gallery of Fluid Motion video winners}

- Lagrangian coherent structures in the flow field of a fluidic oscillator. Moritz Sieber, Florian Ostermann, Kilian Oberleithner, C. Oliver Paschereit, and Rene Woszidlo.

- Shedding light on pilot-wave phenomena. Pierre-Thomas Brun, Dan Harris, Victor Prost, Julio Quintela, and John W. M. Bush.

- Turning on a dime: Asymmetric vortex formation in hummingbird maneuvering flight. Yan Ren, Haibo Dong, Xinyan Deng, and Bret Tobalske.

- Instabilities and fragmentation of drops in 3D. Silvestre Roberto Gonzalez-Avila, Julien Rapet, Claus-Dieter Ohl, and Pjotr Kerssens.

A few weeks after the meeting, we were contacted by the New York Times and, at their request, provided them with the seven winning videos. These were compiled by David Frank and James Gorman into a feature called "When the situation is fluid, sit back and watch," which was posted on the paper's website on December 21 . To see this video, simply search for its title online. ${ }^{1}$

We conclude with sincere thanks to all the contributors to the 2015 GFM as well as to the nine judges. Special thanks are due to Kenneth Kiger, who has served for many years as the institutional memory of the DFD meetings, assuring continuity of GFM traditions. His patient behind-the-scenes support allowed the 2015 GFM committee to appear vaguely competent. We would also like to express gratitude to the meeting co-Chairs, Triantaphyllos Akylas and Thomas Peacock, for their trust and guidance, to Paul Dlug and Mark Doyle of APS for their sustained assistance with all web-related issues, to Peggy Holland of Meetings and More for her deft coordination of activities, and finally to the APS staff for making it all seem effortless.

\footnotetext{
${ }^{1}$ At time of writing, the link is: http://www.nytimes.com/2015/12/21/science/when-the-situation-is-fluid-sitback-and-watch.html.
} 